\title{
Inside Out: From the Brain to the Education
}

\author{
Betsy Ng* \\ National Institute of Education, Nanyang Technological University, Singapore
}

*Corresponding author: Betsy Ng, National Institute of Education, Nanyang Technological University, 1 Nanyang Walk, Singapore 637616, Singapore.
Received Date: September 19, 2019

Published Date: September 24, 2019

\section{Commentary}

Most of the current work in neuroscience focused on identifying the brain basis of learning disabilities, such as developmental dyslexia, but there is a lack of neuroscience research in students' learning needs and learning difficulties in mainstream schools. Do neuroscience findings provide answers to why students are having learning difficulties or why they are not motivated in their studies? Are we able to utilize the existing neuroscience evidence on language comprehension or executive functioning, and translate them into educational settings? The heart of the question is how to apply the existing neuroscience data and translate into the classroom and school setting? To connect how learning takes place within the brain and in a classroom, Thomas et al. [1] summarized the eight interacting learning systems in the brain using a simple thumbnail sketch, which is very relevant to the practitioners and teachers. First, the hippocampus encodes episodic or autobiographical memory. It also consolidates information from short-term to long term memory, as well as spatial memory that enables navigation. Second, the brain connects the information between perceptual information and motor responses, recognizing the complex spatial and temporal patterns which are termed as "concepts". This connecting process within the cortex can take seconds, minutes or hours. Third, the limbic structure further makes connections between stimulus and response which is referred to as "classical conditioning". This connection can occur over seconds or minutes. Fourth, the content-specific systems in the posterior cortex will also be activated, implying the integration of sensory information. Control is in the prefrontal cortex which is involved in planning actions. When a student is in control of a learning task, the prefrontal cortex will be activated, indicating the interaction with the limbic structure (with emotion). Fifth, the reward-based system (also known as dopaminergic value system) operates over seconds or minutes. Learning is a neural process that requires the reinforcement of synaptic functioning and is strongly mediated by dopamine and attentional gain in the frontal cortex
[2]. Sixth, the brain has a procedural learning system for frequent, often unconscious learning tasks. Seventh, the brain can "model" (i.e., observe other people) and acquire skills without much effort. Finally, the brain can take advantage of its neural circuits for constructing new "concepts", thus learning new skills through instruction.

Based on the neuropsychological approach, research interventions such as the three multi-tier approach have been conducted in schools. These three multitier interventions require teachers, school psychologists and educational psychologist to intervene at lower primary schools within the school setting. The three levels are Tier 1 for the whole school and classroom context; Tier 2 for a small group of students, and finally Tier 3 for individual students. The multi-tier framework is a collaborative assessment mechanism to identify students' needs early so as to provide a collaborative, evidence-informed intervention response. Hence, neuroscience data may be indispensable. The trained school psychologist can support the intervention that may overcome or circumvent the students' learning difficulties. However, improved understanding of the neuroscience evidence is still necessary. Despite much of the neuroscience data is on developmental dyslexia or dyscalculia, language mastery or mathematical concepts still pose learning obstacles and challenges for students and teachers, respectively. Then how should we move on from here?

The translation of neuroscience approach into education is still a current debate. Researchers may wrestle with the idea of bringing in electroencephalography (EEG) caps into the classroom to measure students' engagement and emotion. They would collect neuroscience data and then corroborate them with behavioral data, to inform about learning disabilities or difficulties. Together with the behavioral data, the neuroscience findings would give us a better understanding of the brain, indicating the specific areas of brain activation in response to a learning task [2]. However, findings 
from neuroscience research typically draw on small sample sizes and are usually not generalizable.

An example of a neuroscience research is the exploration of language representation and processing of syntactic categories (e.g., noun and verb). Henderson and his colleagues (2016) used a combined approach of eye tracking and functional Magnetic Resonance Imaging (fMRI) to measure the neural foundations of syntax and its effects on language comprehension [3]. They examined the cortical implementation of syntactic computations during native language comprehension on 40 native speakers. Their results revealed that activation in cortical regions are associated with linguistic prediction during comprehension. How do we use such neuroscience findings to inform teachers and practitioners? Are there ways to improve the cortical activations in order for learners to process the syntax of language? Strategies that support learners who are weak in comprehension would likely be more appealing to teachers and practitioners.

It is highly plausible to explore the existing neuroscience findings that could assist in formulating strategies to support teachers. Teachers are pragmatic and intuitive; they do not need a high-end approach to understand their students' needs. In fact, it will be better to educate teachers with the basic neuroscience knowledge, so that their understanding of student's learning difficulty (e.g., in language) is enhanced. The role of neuroscience data is to provide the scientific data or evidence, and to support teachers with relevant teaching strategies for the weaker learners or students with learning difficulties. The existing neuroscience evidence also serves as a basis of getting teachers to understand the rationale of providing the appropriate teaching strategy to support students' learning. In summary, are we able to translate the existing neuroscience data into the classrooms or even at home (as parents)? Education is like a social ecological system. Besides teachers' support for the neuroscience knowledge or approach, without the mindset shift of parents to accept it as well, the translation of neuroscience research into the classroom or learning space seems far-fetched. However, deploying the existing neuroscience data or evidence, it is likely considered the most pragmatic approach in developing appropriate teaching and learning strategies for both teachers and learners, respectively. Likewise, finding a way to contextualize the existing neuroscience data may seem to be another possible solution to bridge the gap between neuroscience and education. Nevertheless, future direction for educational neuroscience research still requires a multidisciplinary team and an interdisciplinary collaborative effort.

\section{Acknowledgement}

None.

\section{Conflict of Interest}

No conflict of interest.

\section{References}

1. Thomas MS, Ansari D, Knowland VC (2019) Annual Research Review: Educational neuroscience: Progress and prospects. Journal of Child Psychology and Psychiatry 60(4): 477-492.

2. Ng B (2018) The neuroscience of growth mindset and intrinsic motivation. Brain Sciences 8(2): 20.

3. Henderson JM, Choi W, Lowder MW, Ferreira F (2016) Language structure in the brain: A fixation-related fMRI study of syntactic surprisal in reading. Neuroimage 132: 293-300.

4. Ng B, Ong AK (2018) Neuroscience and digital learning environment in universities: What do current research tell us? Journal of the Scholarship of Teaching and Learning 18(3): 116-131. 